\title{
Identifying barriers that prevent office-based practitioner referrals for community pharmacist-led diabetes education programs
}

\begin{abstract}
Objective: The primary objective of this study was to determine the barriers preventing office-based practitioner referrals for educational and clinical diabetes services provided by community pharmacists.

Methods: This was a prospective, multi-centered, survey-based study. The top 100 prescribers were identified in three different regions of the Chicagoland area and contacted via fax to complete a 12 -item survey. Data collected included: respondent demographics, knowledge about the role of the clinical specialist pharmacist, knowledge about community pharmacist-led diabetes education programs, types of patients likely to be referred into these programs, and barriers to referrals, if applicable. Completed surveys were interpreted through descriptive and comparative statistics.

Results: Sixteen out of 263 eligiblesurveys were completed and returned (response rate $=6.0 \%$ ). Sixty percent of participants identified lack of knowledge about the service as a barrier to referrals. Forty-four percent identified lack of knowledge on enrollment and lack of follow-up from the pharmacist as other potential barriers. Newly diagnosed and poorly managed patients with diabetes were the patients most likely to be referred by office-based practitioners.
\end{abstract}

Conclusion: Community pharmacists need to provide education to office-based practitioners to help increase referrals and strengthen interprofessional relationships to further enhance patient care.

Keywords: barriers, referrals, community-pharmacists, practitioners, diabetes education programs
Volume 3 Issue 2 - 2015

\author{
Jasmine Mencia ${ }^{1,2}$ Chandni Patel,' Megan \\ Wagner,' Beatrice Drambarean, ${ }^{2}$ Caitlin \\ Malone \\ 'Department of Pharmacy, New Albertsons Inc, USA \\ ${ }^{2}$ Department of Pharmacy Practice, University of Illinois at \\ Chicago College of Pharmacy, USA
}

\section{Correspondence: Jasmine Mencia, New Albertsons Inc. Pharmacies/University of Illinois at Chicago College of Pharmacy, USA, Tel 3024491868,}

Email Jasmine.Mencia@albertsons.com

Received: October 31, 2014| Published: December 04, 2015
Abbreviations: ADA, american diabetes association; CVD, cardiovascular disease; DSME, diabetes self management education; $\mathrm{CS}$, clinical specialist

\section{Introduction}

Diabetes is prevalent in approximately 25.8 million children and adults in the United States, with an additional 79million people estimated to have prediabetes. ${ }^{1}$ In 2007 , the total cost of diagnosed diabetes was $\$ 174$ billion, substantially contributing to the financial burden seen in the United States. ${ }^{1}$ Unfortunately, these numbers are expected to rise, because only $10-20 \%$ of patients with diabetes achieve their therapeutic goals. ${ }^{2}$ Patients with poorly controlled diabetes are at increased risk for cardiovascular disease (CVD). The American Diabetes Association (ADA) has recognized diabetes as a controllable risk factor for CVD; therefore attentive management of blood glucose, blood pressure, and LDL-cholesterol levels is essential and can be achieved through diabetes self management education (DSME) program..$^{3-5}$

DSME programs have become an essential component of diabetes management. Patients participating in DSME have shown improvements in diabetes knowledge, self-care behaviors, clinical outcomes, and quality of life. ${ }^{4}$ Pharmacists can play a significant role in these programs and have been shown to contribute to positive clinical outcomes such as improving glycemic control. ${ }^{6}$ Due to the increasing prevalence of diabetes and the lack of patients reaching recommended therapeutic goals, team-based care models have emerged. ${ }^{5}$ Community pharmacists and general practitioners are increasingly being encouraged to adopt more collaborative approaches, because partnerships in primary care have shown to be effective in improving patient outcomes. ${ }^{7}$

Despite this encouragement of collaborative approaches, a disconnect exists between primary care practitioners and community pharmacists. The purpose of this study was to identify the barriers preventing office-based practitioner referrals to community pharmacist-led diabetes education programs. Secondary objectives determined: if responder demographics influence referrals; if education is needed to improve referrals; the types of patients likely to be referred; and the type of surveys likely to be collected.

\section{Materials and methods}

This was a prospective, multi-centered, survey-based study, approved by the IRB on November 13, 2013, where the top 100 prescribers, from three different regions of the Chicagoland area,were contacted via fax to participate in the study. Practitioners aged 18years and older, working in a primary care office that completed a faxed or online survey (Appendix A) were included in this study. Surveys that were partially completed or completed by practitioners who did not treat patients with diabetes were excluded.

To determine which practitioners should be contacted, a report identifying the top 100 prescribers in three different regions of 
the Chicagoland area was generated using New Albertsons Inc. Pharmacies' computer system. Identified practitioners were then faxed a cover letter and a copy of the survey, and were provided a link to complete the survey electronically. The survey consisted of multiple-choice and select all that apply questions and evaluated the following: practitioner demographics, general views of the clinical specialist (CS), knowledge of the Tools for Living Healthy with Diabetes program, types of patients likely to be referred, and barriers to referral. The CS role was defined as a pharmacist who is trained to counsel and manage patients with chronic disease states and provide appointment-based services outside of workflow.

Surveys were faxed atweeks 0,2 , and 4 beginning on January 3, 2014. Data collection ended at week 6. To incentivize participants, two $\$ 50$ gift cards were raffled and awarded to respondents.

\section{Results}

Three hundred practitioners were identified and contacted via fax to participate in the study. Of the 300 faxes sent, 37 fax numbers were found to be invalid, leaving 263 eligible participants. At the end of the study period, only 16 surveys were completed and returned via fax. No surveys were completed online.

Of the 16 participants, the majority $(31 \%)$ was between the ages of 30 and 39 and reported being in practice for over 20years (44\%). In addition, a majority were medical doctors (69\%). Demographics of practitioners are represented in Table 1.

Table I Respondent demographics and characteristics

\begin{tabular}{|c|c|}
\hline Variable & $\mathrm{n}, \%$ \\
\hline Treat patients with diabetes & 16,100 \\
\hline Males & 8,50 \\
\hline \multicolumn{2}{|l|}{ Age(years) } \\
\hline $20-29$ & 1,6 \\
\hline $30-39$ & 5,31 \\
\hline $40-49$ & 2,12 \\
\hline $50-59$ & 4,25 \\
\hline$\geq 60$ & 2,13 \\
\hline Unknown & 2,13 \\
\hline \multicolumn{2}{|c|}{ Number of years in practice } \\
\hline$<5$ & 4,25 \\
\hline $5-10$ & 2,12 \\
\hline $\mid I-20$ & 3,19 \\
\hline$\geq 20$ & 7,44 \\
\hline \multicolumn{2}{|l|}{ Occupation } \\
\hline MD & 11,69 \\
\hline APN & 4,25 \\
\hline DO & 1,6 \\
\hline
\end{tabular}

Approximately $56 \%$ of the participants were aware of the CS role; however only $18 \%$ were aware that the CS provided appointmentbased services outside of workflow. Furthermore, almost all of the practitioners were unaware of the diabetes education program offered by Jewel-Osco; only 1 out of the 16 respondents knew about the program. Based on study results, newly diagnosed patients and poorly managed patients were the top 2 types of patients likely to be referred by practitioners. Of the 16 respondents, $15(94 \%)$ reported they would refer poorly managed patients and $13(81 \%)$ reported they would refer newly diagnosed patients (Table 2). Other patient groups likely to be referred included those on multiple medications and those on insulin. Only one participant reported they would not refer any patients and this was the same participant who knew about the diabetes education program. When identifying barriers that prevent office-based practitioner referrals (Table 2), it was determined that the top 3 barriers to referral were: lack of knowledge about the service $(56 \%)$, lack of knowledge on how to get patients enrolled (44\%), and lack of follow-up from the pharmacist regarding patient visits (44\%). Other barriers to referral included: fear of lost services, not within the scope of pharmacy, need permission from employer, poor service, and concern about insurance coverage.

Table 2 Respondent barriers to referral and patients likely to be referred

\begin{tabular}{ll}
\hline Variable & Yes (n,\%) \\
\hline Barriers to referral & \\
Fear of lost services & $1,6.3$ \\
Not within scope of pharmacy & $3,18.8$ \\
Patients would not be interested & $3,18.8$ \\
Lack of knowledge about the service & $9,56.3$ \\
Lack of knowledge about enrollment & $7,43.8$ \\
Lack of follow-up from the pharmacist & $7,43.8$ \\
Other & $3,18.8$ \\
Patients likely to be referred & \\
Newly diagnosed & $13,81.3$ \\
Poorly managed & $15,93.8$ \\
On multiple medications & $8,50.0$ \\
On insulin & $9,56.3$ \\
Certain age groups & $3,18.8$ \\
Certain gender & $3,18.8$
\end{tabular}

Based on statistical analyses (using a Fisher's Exact Test, $\alpha=0.05$ ), responder demographics did not influence barriers to referral; however, they did influence the types of patients likely to be referred. All practitioners practicing for 11 to 20years responded that they would refer newly diagnosed patients to this type of service, whereas there were some discrepancies within the other years in practice groups $(\mathrm{p}=0.002)$. In addition, only advanced practice nurses responded they would refer certain age groups to this type of service, whereas other professions did not $(\mathrm{p}=0.02)$.

\section{Discussion}

Despite the small sample size, this study included a diverse 
group of respondents and provided insight on barriers to office-based referrals. Based on results, it is evident office-based practitioners need to be educated on community pharmacist led diabetes services and this education should be provided by community pharmacists via an office in-service or doctor visit to help increase referrals. Inservices and office visits should focus on providing education about the service, how patients can be enrolled, as well as, address how the pharmacist will keep the practitioner informed on the patient's progress, since these three areas were identified as the top barriers to referral. Furthermore, it appears pharmacists may have the greatest opportunity to collaborate with practitioners when it comes to newly diagnosed patients and poorly managed patients, since these were the top two types of patients likely to be referred. This type of collaboration will strengthen inter professional relationships and further enhance patient care.

\section{Limitations}

Out of the 263 potential responders, only 16 completed surveys were faxed and returned leaving a small sample size to be analyzed. Potential reasons for the sample being so small may be due to the fact that only a select group of practitioners were contacted from a specific geographic region, in this case the Chicagoland area. Furthermore, sample size may have been limited due to the fact that no online surveys were completed, faxes were sent right after the Christmas/ New Year holiday, and the study duration was short (only 6weeks long). All of these factors most likely contributed to the low response rate of $6 \%$, which was another limitation of this study.

\section{Conclusion}

In conclusion, the disconnect that exists between office-based practitioners and community pharmacists appears to be mainly due to lack of knowledge on the practitioners behalf about the services available in the community and how to enroll patients into these services. Community pharmacists need to provide this information to offices through educational in-services or discussions to increase collaborative team based approaches, further enhancing patient care.

\section{Acknowledgements}

The author would like to acknowledge New Albertsons Inc. Pharmacies and the University of Illinois at Chicago College of Pharmacy for their contributions to this research.

\section{Conflict of interest}

Author declares that there is no conflict of interest.

\section{References}

1. Center for Disease Control and Prevention. National Diabetes Fact Sheet. 2011.

2. Saydah SH, Fradkin J, Cowie CC. Poor control of risk factors for vascular disease among adults with previously diagnosed diabetes. JAMA. 2004;291(3):335-342.

3. Yu J, Shah BM, Ip EJ, et al. A Markov model of the cost-effectiveness of pharmacist care for diabetes in prevention of cardiovascular diseases: evidence from Kaiser Permanente Northern California. J Manag Care Pharm. 2013;19(2):102-114.

4. American Diabetes Association. Standards of Medical Care in Diabetes-2011. Diabetes Care. 2011;34 Suppl 1:S11-S61.

5. Gerber BS, Rapacki L, Castillo A, et al. Design of a trial to evaluate the impact of clinical pharmacists and community health promoters working with African-Americans and Latinos with diabetes. BMC Public Health. 2012; $12: 891$

6. Iyer R, Coderre P, McKelvey T, et al. An employer-based, pharmacist intervention model for patients with type 2 diabetes. Am J Health Syst Pharm. 2010;67(4):312-316.

7. Van C, Costa D, Abbott P, et al. Community pharmacist attitudes towards collaboration with general practitioners: development and validation of a measure and a model. BMC Health Serv Res. 2012;12:320. 